Article

\title{
Dynamic Collection Strategy and Coordination of a Remanufacturing Closed-Loop Supply Chain under Uncertainty
}

\author{
Zongsheng Huang ${ }^{1,+}$, Jiajia Nie ${ }^{2,+}$ and Sang-Bing Tsai ${ }^{3,4,5, *,+}$ \\ 1 School of Economics and Management, Shanghai Maritime University, Shanghai 201306, China; \\ zshuang@shmtu.edu.cn \\ 2 School of Economics and Management, Southwest Jiaotong University, Chengdu 610031, China; \\ niejiajia@home.swjtu.edu.cn \\ 3 Business and Law School, Foshan University, Foshan 528000, China \\ 4 Zhongshan Institute, University of Electronic Science and Technology of China, Zhongshan 528400, China \\ 5 Economics and Management College, Civil Aviation University of China, Tianjin 300300, China \\ * Correspondence: sangbing@hotmail.com \\ $\dagger$ The authors are ordered alphabetically and share equal contribution.
}

Academic Editor: Andrea Appolloni

Received: 21 March 2017; Accepted: 19 April 2017; Published: 26 April 2017

\begin{abstract}
This paper aims to investigate the uncertainty factors that impact the collection strategy of a remanufacturing closed-loop supply chain. The basis of this type of supply chain is the collection of used products, without which the remanufacturing of new products would be out of the question. In this paper, we considered the effect of stochastic disturbance on the collection process in a closed-loop supply chain consisting of a manufacturer and a retailer, in which the manufacturer is responsible for collecting the used products. Considering stochastic disturbance, in this research, we proposed a stochastic collection model, and derived the feedback control strategies for both the manufacturer and retailer. Next, we investigated the evolutionary path and probability distribution of the stochastic return rate. Finally, we proposed a contract to coordinate the decentralized closed-loop supply chain. The results showed that the manufacturer would improve the level of return effort as the intensity of stochastic disturbance increases. However, the disturbance would not affect the pricing strategies of the supply chain members. As a result of the stochastic disturbance, the return rate always hovered around the expected return rate, while the expectation and variance of the return rate remained stable from a long-term perspective. The expected value and variance of the return rate could decrease or increase with time, depending on the value of the initial return rate of the system.
\end{abstract}

Keywords: closed-loop supply chain; coordination contract; feedback control strategy; stochastic differential game; green supply chain

\section{Introduction}

Remanufacturing of used products consumes fewer natural materials and less energy than conventional manufacturing, thus reducing the generation of waste and carbon emissions [1]. For instance, remanufacturing photocopiers can save $20-70 \%$ of the natural materials and energy needed, and reduce waste by $35-50 \%$ as compared to conventional manufacturing [2]. It is obvious that reduced consumption of raw materials and energy is conducive to the reduction of carbon emissions. Additionally, remanufacturing can lower production cost for the manufacturer. Consequently, an increasing number of manufacturers have begun to execute remanufacturing strategies, including HP, Lenovo, Apple, and Xerox, among others [3,4]. 
The basis of a remanufacturing closed-loop supply chain (CLSC) is the collection of used products, without which the remanufacturing of new products is out of the question. Shaharudin et al. [5] has found that the extent of product collection has a positive significant effect on the adoption of CLSC activities. However, the collection process is quite complex as a result of high uncertainty regarding the quantity, quality, and timing of returns [6-8]. The customers' willingness to return their used-product also contributes to the complexity of the collection problem $[9,10]$. Any manufacturer who aims to collect the used products must cope with this uncertainty and complexity.

In the study of CLSCs, most of the previous research, such as the work of Savaskan et al. [11], and Savaskan and Van Wassenhove [3], adopted the static return rate model. However, this model ignores the dynamic character of the activities in which manufacturers must invest in order to collect used products. For instance, manufacturers face marketing expenditures, such as advertising, designed to increase the environmental awareness of customers and improve their knowledge about the used product return policies. Manufacturers also must invest in operational activities such as creating and maintaining recycling facilities, collection arrangements, reverse logistics, reprocessing strategies, and so on. The marketing activities and operational activities both have dynamic and long term effects, which means that the return rate reflects the whole investment history [12]. In this way, the investment and return characteristics for a CLSC are similar to those presented in the dynamic advertising problem [13]. To study the dynamic return problem, we developed a stochastic differential game model similar to the one presented by De Giovanni and Zaccour [12]. The model simultaneously considered the dynamic effect of the collection process and the stochastic factors during the collection activities. We then applied the optimal control method to solve the model.

In the field of marketing, optimal control models are used widely to cope with the dynamic nature of advertising as well as with dynamic pricing problems. Examples include the advertising goodwill model [13], advertising sales volume model [14], and market growth model [15] along with its extensions [16-19]. However, to the best of our knowledge, there has been little research that utilizes the dynamic control model to investigate the return problem in the CLSC area. De Giovanni and Zaccour [12] and Huang and Nie [19] were two of the few attempts made in this direction.

In order to investigate the optimal control strategy for the manufacturer and retailer in a CLSC, the stochastic differential game model that we developed takes into account stochastic disturbance during the collection process. The used products are collected by the manufacturer, the remanufactured products do not differ in any way from the new products manufactured from raw materials, and both types of products are sold at the same price in the same markets. The manufacturer facing the stochastic disturbance in the collection process is the leader in the CLSC approach; thus, the supply chain members are playing the Stackelberg differential game with the manufacturer as channel leader. The manufacturer simultaneously determines the wholesale price as well as the level of the effort for collecting the used products, and then the retailer determines the retail price. The differential game is solved by the Hamilton-Jacobi-Bellman (HJB) equation method.

The main contribution of this work seeks to implement a differential game model to investigate the optimal control strategies for the CLSC members in a stochastic dynamic scenario, and study how the stochastic factors in the collection process would affect the return rate of the CLSC system. Furthermore, the paper proposes a coordination contract to boost the efficiency of the decentralized CLSC.

As the importance of remanufacturing has increased, so has the amount of research dealing with the operation of a CLSC, such as the work of Savaskan et al. [11], and Savaskan and Van Wassenhove [3]. We refer the reader to Atasu et al. [20], Govindan et al. [21] and Govindan and Soleimani [22] for comprehensive reviews of CLSC operations. Since we focused on the product collection problem in a CLSC with a dynamic and stochastic setting, our research is related to two streams of the available literature.

The first stream includes papers that concentrated on the reverse channel management problem in various CLSC scenarios. Savaskan et al. [11] modeled three common reverse channel options for CLSCs: the manufacturer's collection channel, the retailer's collection channel, and a third-party 
collection channel. Comparing the three types of channels, they found that the retailer's collection process might be the optimal reverse channel for the CLSC. Savaskan and Van Wassenhove [3] studied the reverse channel design problem further by extending the model into the scenario of competing retailers. Huang et al. [23] considered a CLSC with a dual recycle channel in which the manufacturer sells products via the retailer in the forward chain, while the retailer and the third party simultaneously collect used products in the reverse chain. They found that the dual recycling channel dominated the single recycling channel when the reverse channel competition is not very strong. Zhang et al. [24] considered the CLSC problem in a dual channel supply chain with the manufacturer selling products via direct Internet channels as well as indirect retailer channels. De Giovanni and Zaccour [25] studied the collection outsourcing choice of the manufacturer in a CLSC, where the retailer or the third-party firm can undertake the outsourcing task. These papers mainly discussed the reverse channel design problem with a single member in the supply chain to undertake the collection task [26-30].

Subramanian et al. [31] considered the effect of extended producer responsibility (EPR) on a remanufacturing supply chain. Jacobs and Subramanian [32] also considered the EPR programs, and they attempted to share the EPR responsibility within the supply chain. Jena and Sarmah [33] studied the co-operation and competition issues in a CLSC that consisted of two manufacturers who compete for selling as well as collecting the used products. Ma et al. [34] investigated various cooperative models among the different parties in a CLSC consisting of a single manufacturer, a single retailer, and two recyclers, with the main focus on how the cooperative strategies might affect the CLSC system. These papers mainly looked at ways to allocate the collection activity in the supply chain to improve the collection efficiency. Our paper contributes to this stream by utilizing the differential game model to study the collecting strategies in a decentralized CLSC.

The second stream of research related to our model is the investigation of used product acquisition in a centralized or decentralized system. Guide and Wassenhove [35] considered the used product collection problem in the face of uncertain quality. Nakashima et al. [36] studied the optimal control problem in remanufacturing systems. Fallah et al. [37] studied the competition between two CLSCs in an uncertain environment, with the competition including retail prices in the forward channel, and incentives paid to consumers in the reverse channel. Their major focus was on closed-loop network design decisions, using fuzzy set theory to handle the uncertainties. This area of research focused mainly on the issues of product collection in terms of quality or timing uncertainty, but the collection activities had their dynamic nature as well $[12,38,39]$.

De Giovanni and Zaccour [12] proposed a dynamic collection model that utilized a differential game method to characterize the feedback equilibrium of a CLSC with one manufacturer and one retailer. A cost and revenue-sharing contract was proposed. They found that the retailer who acts as the leader always prefers the contract, while the manufacturer only prefers the contract under particular conditions. Huang and Nie [19] also proposed a dynamic collection model that captured the dynamic characteristics of the collection process. They derived the open-loop control strategies of both manufacturer and retailer in a manufacturer-led Stackelberg game. These two papers are most closely related to our research. However, we took the stochastic disturbance into consideration and focused on the effect of stochastic factors on the control strategies of the manufacturer and retailer. Further, we studied the coordination problem of the CLSC, and the results showed that the stochastic supply chain can be coordinated by the proposed contract.

The remainder of this paper is organized as follows. Section 2 presents our modeling framework. Sections 3 and 4 show how we derived the feedback control strategies for the integrated channel and the decentralized channel, respectively. Section 5 explores the coordination problem in the decentralized CLSC. Section 6 concludes the paper.

\section{The Model}

The product return model we adopted is similar to the ones used by Huang and Nie [19] and De Giovanni and Zaccour [12], which supposed that the real time return rate has a cumulative effect. 
The return rate in our model is formulated as a continuous and weighted average of past return activities, with a decaying weighting function. Defining return rate $\tau(t)$ as the state variable, and the return effort $A(t)$ as the control variable, the dynamic return process is captured by the following formula:

$$
d \tau(t)=(\rho A(t)-\delta \tau(t)) d t
$$

where $\delta$ is the decaying factor, and $\rho$ is the extent of the impact of return activities on the return rate.

Equation (1) captures the dynamics of the product return process, but it does not consider random disturbance in the process. Actually, there are many factors that can disturb the return rate, such as the wide geographic spread of used products to be returned, and the complexity of reverse transportation [40]. Considering stochastic disturbance, we model the return rate $\tau(t)$ by the Itô equation as shown:

$$
d \tau(t)=(\rho A(t)-\delta \tau(t)) d t+\sigma(\tau(t)) d z(t), \tau(0)=\tau_{0},
$$

where $\sigma(\tau(t))$ is a variance term, $z(t)$ is a standard Wiener process, $\tau_{0}$ is the initial return rate, and $\tau_{0} \in[0,1)$. The model in Equation (2) is intuitive insofar as the term $(\rho A(t)-\delta \tau(t)) d t$ represents the net influence of return effort on the return rate, which is deterministic, and the term $\sigma(\tau(t)) d z(t)$ represents the stochastic disturbance of the random factors on the return rate, which is indeterminate.

It should be noted that the return rate must satisfy $0 \leq \tau \leq 1$. As a result, $A$ and $\sigma(\tau)$ should be continuous functions, and they should satisfy the Lipschitz conditions on each closed subinterval of $(0,1)$. In addition, $A(\tau) \geq 0$ when $\tau \in[0,1]$, and

$$
\sigma(\tau)>0, \text { when } \tau \in(0,1) \text { and } \sigma(0)=0
$$

With this condition, the drift at $\tau=0$ is positive as long as $\rho A(0)-\delta \tau(0)>0$, which can be met easily when $\tau(0)$ is small enough. Since we consider the return rate is not able to reach 1 , we ignore the case that $\tau=1$. Similar to the work of Prasad and Sethi [41], we let $\sigma(\tau)=\sigma \sqrt{\tau}$ to simplify the complexity in the study, which satisfies the condition when $\sigma$ is a positive constant.

The cost function of return activities for the manufacturer is assumed by $k A(t)^{2} / 2$, where $k$ is the return cost coefficient. In reality, the used products could be distributed widely across the market, which could cause a comparatively high return cost coefficient for the manufacturer.

The per unit cost of manufacturing a new product unit from raw materials or from used materials is represented by $c_{m}$ and $c_{r}$, respectively. We assume $c_{r}<c_{m}$, which indicates that remanufacturing is profitable to the manufacturer. Let $\Delta=c_{m}-c_{r}$ to denote the cost savings from remanufacturing.

Utilizing $p(t)$ to denote the retail price, which is controlled by the retailer, the demand rate for the product at time $t$ is calculated as follows:

$$
D(t)=\phi-\beta p(t)
$$

where $\phi$ represents the market potential capacity, and $\beta$ represents the price sensitivity coefficient.

The supply chain consists of one manufacturer, the Stackelberg leader, and one retailer, the Stackelberg follower. The time preference rate of supply chain members is denoted by $r$ and the planning period is $[0,+\infty)$. Controlling the wholesale price $w(t)$ and the collection effort $A(t)$, the objective function of the manufacturer is formulated as follows:

$$
\max _{w>0, A>0}\left\{J_{m}=E \int_{0}^{\infty}\left[\left(w-c_{m}+\Delta \tau\right)(\phi-\beta p)-\frac{k}{2} A^{2}\right] d t\right\} .
$$

Controlling the retail price $p(t)$, the objective function of the retailer is formulated as shown:

$$
\max _{p>0}\left\{J_{r}=E \int_{0}^{\infty}[(p-w)(\phi-\beta p)] d t\right\} .
$$


The supply chain members seek to maximize their expected discounted profit stream subject to the system dynamics in Equation (2). We adopted the Hamilton-Jacobi-Bellman (HJB) equation method to resolve the stochastic differential game presented above.

\section{Benchmark Model: The Integrated Channel}

In this section, we consider the integrated channel scenario in which the retailer is not involved in the decision making process, and the manufacturer behaves as the central decision maker who makes the pricing and collecting decisions. This provides the benchmark results for our analysis.

The stochastic return rate is given by Equation (2), and the demand rate is given by Equation (3). In the integrated channel scenario, there is no wholesale price, and the objective of the central decision maker is formulated as shown:

$$
\max _{p>0, A>0}\left\{J_{C}=E \int_{0}^{\infty} e^{-r t}\left[\left(p-c_{m}+\Delta \tau\right)(\phi-\beta p)-\frac{k}{2} A^{2}\right] d t\right\} .
$$

The central decision maker simultaneously decides both the retail price $p(t)$ as well as the level of collection effort $A(t)$. The Hamilton-Jacobi-Bellman (HJB) equation is formulated as follows:

$$
r V_{C}=\max _{p, A}\left\{\left(p-c_{m}+\Delta \tau\right)(\phi-\beta p)-\frac{k}{2} A^{2}+V_{C}{ }^{\prime}(\tau)(\rho A-\delta \tau)+\frac{\sigma^{2}}{2} V^{\prime \prime} C(\tau)\right\}
$$

in which $V_{C}^{\prime}(\tau)=d V_{C}(\tau) / d \tau$, and $V^{\prime \prime} C(\tau)=d^{2} V_{C}(\tau) / d \tau^{2}$.

Applying the first-order condition to Equation (7) provides the feedback strategies of the central decision maker:

$$
A^{*}=\frac{\rho}{k} V^{\prime}{ }_{C}, p^{*}=\frac{\phi+\beta c_{m}-\beta \Delta \tau}{2 \beta} .
$$

Taking Equation (8) back into Equation (7), we then have the following equation:

$$
r V_{C}=\frac{1}{4} \beta \Delta^{2} \tau^{2}+\left(\frac{1}{2} \Delta\left(\phi-\beta c_{m}\right)-\delta V_{C}^{\prime}+\frac{1}{2} \sigma^{2} V^{\prime \prime} C\right) \tau+\frac{\left(\phi-\beta c_{m}\right)^{2}}{4 \beta}+\frac{1}{2} \frac{\rho^{2}}{k}\left(V_{C}^{\prime}\right)^{2} .
$$

Similar to the approach used by Erickson [42], we conjecture the value function is $V_{C}=\frac{1}{2} e_{1} \tau^{2}+$ $e_{2} \tau+e_{3}$, in which $e_{i}(i=1,2,3)$ are coefficients that need to be determined. We obtain $V^{\prime}{ }_{C}=e_{1} \tau+e_{2}$ and $V^{\prime \prime}{ }_{C}=e_{1}$ directly. Inserting the conjectured value function and its derivations into Equation (9), we get the following result:

$$
r V_{c}=\left\{\begin{array}{l}
{\left[\frac{1}{4} \beta \Delta^{2}+e_{1}\left(\frac{\rho^{2} e_{1}}{k}-\delta\right)-\frac{\rho^{2} e_{1}^{2}}{2 k}\right] \tau^{2}+\left[\frac{1}{2} \Delta\left(\phi-\beta c_{m}\right)+e_{2}\left(\frac{\rho^{2} e_{1}}{k}-\delta\right)+\frac{1}{2} \sigma^{2} e_{1}\right] \tau} \\
+\frac{\left(\phi-\beta c_{m}\right)^{2}}{4 \beta}+\frac{1}{2} \frac{\rho^{2}}{k} e_{2}^{2}
\end{array}\right\} .
$$

Based on the equal coefficient principle, we have the following equations:

$$
\left\{\begin{array}{l}
\frac{r}{2} e_{1}=\frac{1}{4} \beta \Delta^{2}+e_{1}\left(\frac{\rho^{2} e_{1}}{k}-\delta\right)-\frac{\rho^{2} e_{1}^{2}}{2 k} \\
r e_{2}=\frac{1}{2} \Delta\left(\phi-\beta c_{m}\right)+e_{2}\left(\frac{\rho^{2} e_{1}}{k}-\delta\right)+\frac{1}{2} \sigma^{2} e_{1} . \\
r e_{3}=\frac{\left(\phi-\beta c_{m}\right)^{2}}{4 \beta}+\frac{1}{2} \frac{\rho^{2}}{k} e_{2}^{2}
\end{array}\right.
$$

The coefficients are solved as shown:

$$
\left\{\begin{array}{l}
e_{1}=\frac{(r+2 \delta) k \pm \sqrt{(r+2 \delta)^{2} k^{2}-2 \beta k \rho^{2} \Delta^{2}}}{2 \rho^{2}} \\
e_{2}=\frac{1}{2} \frac{\left[\left(\phi-\beta c_{m}\right) \Delta+\sigma^{2} e_{1}\right] k}{(r+\delta) k-\rho^{2} e_{1}} \\
e_{3}=\frac{k\left(\phi-\beta c_{m}\right)^{2}+2 \rho^{2} \beta e_{2}^{2}}{4 \beta k r}
\end{array} .\right.
$$


Assume that $(r+2 \delta)^{2} k^{2}-2 \beta k \rho^{2} \Delta^{2}>0$ to ensure that $e_{1}$ is real. Now we have $e_{1}>0$. To assure the solution is meaningful in practice, $e_{2}$ should be positive, or else the collection effort level might be negative when the return rate $\tau$ is small. To make $e_{2}>0$, we have the following:

$$
\sqrt{(r+2 \delta)^{2} k^{2}-2 \beta k \rho^{2} \Delta^{2}}>r k
$$

and

$$
(r+\delta) k-\rho^{2} e_{1}=\frac{r k \mp \sqrt{(r+2 \delta)^{2} k^{2}-2 \beta k \rho^{2} \Delta^{2}}}{2} .
$$

Obviously, $r k+\sqrt{(r+2 \delta)^{2} k^{2}-2 \beta k \rho^{2} \Delta^{2}}>0$, and $r k-\sqrt{(r+2 \delta)^{2} k^{2}-2 \beta k \rho^{2} \Delta^{2}}<0$. In other words, when

$$
e_{1}=\frac{(r+2 \delta) k-\sqrt{(r+2 \delta)^{2} k^{2}-2 \beta k \rho^{2} \Delta^{2}}}{2 \rho^{2}},
$$

then $(r+\delta) k-\rho^{2} e_{1}>0, e_{2}>0$, and $A>0$. In addition, when

$$
e_{1}=\frac{(r+2 \delta) k+\sqrt{(r+2 \delta)^{2} k^{2}-2 \beta k \rho^{2} \Delta^{2}}}{2 \rho^{2}}
$$

we have $(r+\delta) k-\rho^{2} e_{1}<0$ and $e_{2}<0$. Thus, when the return rate is small, the collection effort level $A$ may be positive. As a result, we abandon the solution concerning $e_{1}$.

Utilizing the superscript $C$ to denote the scenario of integrated channels, the equilibrium strategies are summarized in the following proposition.

Proposition 1. For the integrated channel, assuming that $2 \delta(r+\delta) k-\beta \rho^{2} \Delta^{2}>0$, we can show:

(1) The optimal retail price control strategy of the central decision maker is given by:

$$
p^{C *}=\frac{\phi+\beta c_{m}-\beta \Delta \tau}{2 \beta}
$$

(2) The optimal collection effort level control strategy is given by:

$$
A^{C *}=\frac{\rho}{k}\left(e_{1} \tau+e_{2}\right)
$$

(3) The value function of the integrated channel is calculated by

$$
V^{C *}=\frac{1}{2} e_{1} \tau^{2}+e_{2} \tau+e_{3}(1)
$$

where

$$
\left\{\begin{array}{l}
e_{1}=\frac{(r+2 \delta) k-\sqrt{(r+2 \delta)^{2} k^{2}-2 \beta k \rho^{2} \Delta^{2}}}{2 \rho^{2}} \\
e_{2}=\frac{1}{2} \frac{\left[\left(\phi-\beta c_{m}\right) \Delta+\sigma^{2} e_{1}\right] k}{(r+\delta) k-\rho^{2} e_{1}} \\
e_{3}=\frac{k\left(\phi-\beta c_{m}\right)^{2}+2 \rho^{2} \beta e_{2}{ }^{2}}{4 \beta k r}
\end{array} .\right.
$$

Proposition 1 indicates the optimal feedback strategies as well as the profit level of the integrated channel. The assumption $2 \delta(r+\delta) k-\beta \rho^{2} \Delta^{2}>0$, which can be rewritten as $k>\beta \Delta^{2} \rho^{2} /(2 \delta(r+\delta))$, means the collection cost coefficient $k$ could not be too small in the model, otherwise the central decision maker would collect all the used products. However, this cannot be true in practice because 
the used products may be spread widely across geographic areas. There is a similar result for the decentralized channel.

\section{Model Analysis: The Decentralized Channel}

In this section, we will seek first to identify the optimal control strategies of the manufacturer and the retailer, and then analyze the evolutionary path as well as the probability distribution of the return rate.

\subsection{Feedback Stackelberg Equilibrium}

The decision sequence of this game is that the manufacturer first chooses the wholesale price $w(t)$ as well as the return effort level $A(t)$, and then the retailer chooses the retail price $p(t)$. The value functions of the manufacturer and the retailer are denoted by $V_{r}$ and $V_{m}$, respectively. The Hamilton-Jacobi-Bellman (HJB) equations of the supply chain members are formulated as follows:

$$
\begin{gathered}
r V_{r}=\max _{p}\left\{(p-w)(\phi-\beta p)+V_{r}^{\prime}(\tau)(\rho A-\delta \tau)+\frac{\sigma^{2}}{2} \tau V^{\prime \prime}{ }_{r}(\tau)\right\}, \\
r V_{m}=\max _{w, A}\left\{\left(w-c_{m}+\Delta \tau\right)(\phi-\beta p)-\frac{k}{2} A^{2}+V_{m}{ }^{\prime}(\tau)(\rho A-\delta \tau)+\frac{\sigma^{2}}{2} \tau V_{m}{ }^{\prime \prime}(\tau)\right\},
\end{gathered}
$$

where $V_{i}^{\prime}(\tau)=d V_{i}(\tau) / d \tau, V_{i}^{\prime \prime}(\tau)=d^{2} V_{i}(\tau) / d \tau^{2}$, and $i=m, r$. According to the backward induction method, first we resolve the decision problem of the retailer. From Equation (16), we can obtain the reaction function of the retailer:

$$
p^{*}=\frac{\phi+\beta w}{2 \beta} .
$$

Taking the reaction of the retailer into consideration, the HJB function of the manufacturer becomes:

$$
r V_{m}=\max _{w, A}\left\{\left(w-c_{m}+\Delta \tau\right) \frac{(\phi-\beta w)}{2}-\frac{k}{2} A^{2}+V_{m}^{\prime}(\tau)(\rho A-\delta \tau)+\frac{\sigma^{2}}{2} \tau V^{\prime \prime}{ }_{m}(\tau)\right\} .
$$

From the first-order condition, we can obtain the optimal feedback strategies of the manufacturer:

$$
A^{*}=\frac{\rho}{k} V_{m}^{\prime}, w^{*}=\frac{\phi+\beta c_{m}-\beta \Delta \tau}{2 \beta} .
$$

Taking Equation (19) back into the reaction of the retailer, we can obtain the optimal strategy:

$$
p^{*}=\frac{3 \phi+\beta c_{m}-\beta \Delta \tau}{4 \beta} .
$$

Substituting Equations (19) and (20) into Equations (15) and (16), we get the following results:

$$
\begin{aligned}
& r V_{r}=\frac{1}{16} \beta \Delta^{2} \tau^{2}+\left(\frac{1}{8} \Delta\left(\phi-\beta c_{m}\right)-\delta V^{\prime}{ }_{r}+\frac{1}{2} \sigma^{2} V^{\prime \prime}{ }_{r}\right) \tau+\frac{\left(\phi-\beta c_{m}\right)^{2}}{16 \beta}+\frac{\rho^{2} V^{\prime}{ }_{r} V_{m}^{\prime}}{k} \\
& r V_{m}=\frac{1}{8} \beta \Delta^{2} \tau^{2}+\left(\frac{1}{4} \Delta\left(\phi-\beta c_{m}\right)-\delta V^{\prime}{ }_{m}+\frac{1}{2} \sigma^{2} V^{\prime \prime}{ }_{m}\right) \tau+\frac{\left(\phi-\beta c_{m}\right)^{2}}{8 \beta}+\frac{\rho^{2}}{2 k}\left(V^{\prime}{ }_{m}\right)^{2} .
\end{aligned}
$$

Again following the work of Erickson [42], we conjecture the value functions of the manufacturer and the retailer are as follows:

$$
\left\{\begin{array}{l}
V_{r}=\frac{1}{2} g_{1} \tau^{2}+g_{2} \tau+g_{3} \\
V_{m}=\frac{1}{2} f_{1} \tau^{2}+f_{2} \tau+f_{3}
\end{array},\right.
$$


where $g_{i}$ and $f_{i}(i=1,2,3)$ are coefficients to be determined. Inserting Equation (22) and its derivations into Equation (21), we can obtain the equations to be solved for the coefficients defined in Equation (22):

$$
\begin{gathered}
\left\{\begin{array}{l}
r \frac{1}{2} f_{1}=\frac{1}{8} \beta \Delta^{2}+f_{1}\left(\frac{\rho^{2} f_{1}}{k}-\delta\right)-\frac{\rho^{2} f_{1}^{2}}{2 k} \\
r f_{2}=\frac{1}{4} \Delta\left(\phi-\beta c_{m}\right)+f_{2}\left(\frac{\rho^{2} f_{1}}{k}-\delta\right)+\frac{1}{2} \sigma^{2} f_{1}, \\
r f_{3}=\frac{\left(\phi-\beta c_{m}\right)^{2}}{8 \beta}+\frac{1}{2} \frac{\rho^{2}}{k} f_{2}^{2}
\end{array}\right. \\
\left\{\begin{array}{l}
r \frac{1}{2} g_{1}=\frac{1}{16} \beta \Delta^{2}+g_{1}\left(\frac{\rho^{2} f_{1}}{k}-\delta\right) \\
r g_{2}=\frac{1}{8} \Delta\left(\phi-\beta c_{m}\right)+g_{2}\left(\frac{\rho^{2} f_{1}}{k}-\delta\right)+\frac{1}{2} \sigma^{2} g_{1}+\frac{\rho^{2}}{k} g_{1} f_{2}, \\
r g_{3}=\frac{\left(\phi-\beta c_{m}\right)^{2}}{16 \beta}+\frac{\rho^{2}}{k} g_{2} f_{2}
\end{array}\right.
\end{gathered}
$$

where Equation (23) shows the undetermined coefficient equations of the manufacturer, and Equation (24) shows the equations of the retailer. The coefficients can be solved from Equations (23) and (24) as follows:

$$
\left\{\begin{array} { l } 
{ f _ { 1 ( 1 , 2 ) } = \frac { ( r + 2 \delta ) k \pm \sqrt { ( r + 2 \delta ) ^ { 2 } k ^ { 2 } - \beta k \Delta ^ { 2 } \rho ^ { 2 } } } { 2 \rho ^ { 2 } } } \\
{ f _ { 2 } = \frac { k } { 4 } \frac { \Delta ( \phi - \beta c _ { m } ) + 2 \sigma ^ { 2 } f _ { 1 } } { ( r + \delta ) k - \rho ^ { 2 } f _ { 1 } } } \\
{ f _ { 3 } = \frac { k ( \phi - \beta c _ { m } ) ^ { 2 } + 4 \beta \rho ^ { 2 } f _ { 2 } ^ { 2 } } { 8 \beta k r } }
\end{array} \left\{\begin{array}{l}
g_{1}=\frac{1}{8} \frac{\beta k \Delta^{2}}{(r+2 \delta) k-2 \rho^{2} f_{1}} \\
g_{2}=\frac{1}{8} \frac{\Delta k\left(\phi-\beta c_{m}\right)+4 k \sigma^{2} g_{1}+8 \rho^{2} g_{1} f_{2}}{(r+\delta) k-\rho^{2} f_{1}} \\
g_{3}=\frac{k\left(\phi-\beta c_{m}\right)^{2}+16 \beta \rho^{2} g_{2} f_{2}}{16 \beta k r}
\end{array} .\right.\right.
$$

It is easy to see that as long as $f_{1}$ is solved, the other coefficients can then be solved.

Assume that $(r+2 \delta)^{2} k^{2}-\beta k \rho^{2} \Delta^{2}>0$ to ensure that $f_{1}$ is real. Then, we have $f_{1}>0$. To assure the solution is meaningful in practice, $f_{2}$ should be positive, or else the return effort may be negative when the return rate $\tau$ is small, which obviously would not conform to reality. To make $f_{2}>0$, we have,

$$
(r+2 \delta)^{2} k^{2}-\beta k \Delta^{2} \rho^{2}>r^{2} k^{2}
$$

and

$$
(r+\delta) k-\rho^{2} f_{1}=\frac{r k \mp \sqrt{(r+2 \delta)^{2} k^{2}-\beta k \Delta^{2} \rho^{2}}}{2} .
$$

Obviously, $r k+\sqrt{(r+2 \delta)^{2} k^{2}-\beta k \Delta^{2} \rho^{2}}>0$, and $r k-\sqrt{(r+2 \delta)^{2} k^{2}-\beta k \Delta^{2} \rho^{2}}<0$. In other words, when

$$
f_{1}=\frac{(r+2 \delta) k-\sqrt{(r+2 \delta)^{2} k^{2}-\beta k \Delta^{2} \rho^{2}}}{2 \rho^{2}},
$$

we have $(r+\delta) k-\rho^{2} f_{1}>0, f_{2}>0$, and $A>0$.

When

$$
f_{1}=\frac{(r+2 \delta) k+\sqrt{(r+2 \delta)^{2} k^{2}-\beta k \Delta^{2} \rho^{2}}}{2 \rho^{2}},
$$

we have $(r+\delta) k-\rho^{2} f_{1}<0$, and $f_{2}<0$. In this case, the return effort $A$ may be negative for some small return rates. To guarantee that the return effort is positive, we abandon the larger solution for $f_{1}$. The following proposition characterizes the feedback equilibrium strategies for the manufacturer and retailer. 
Proposition 2. For the decentralized channel, assuming that $4 \delta(r+\delta) k-\beta \Delta^{2} \rho^{2}>0$, we can show:

(1) The optimal wholesale price control strategy and optimal return effort control strategy of the manufacturer is given by

$$
w^{*}=\frac{\phi+\beta c_{m}-\beta \Delta \tau}{2 \beta}, A^{*}=\frac{\rho}{k}\left(f_{1} \tau+f_{2}\right) ;
$$

(2) The optimal retail price control strategy of the retailer is given by

$$
p^{*}=\frac{3 \phi+\beta c_{m}-\beta \Delta \tau}{4 \beta} ;
$$

(3) The value functions of both manufacturer and retailer are calculated as

$$
V_{r}^{*}=\frac{1}{2} g_{1} \tau^{2}+g_{2} \tau+g_{3}, V_{m}^{*}=\frac{1}{2} f_{1} \tau^{2}+f_{2} \tau+f_{3},
$$

where

$$
\left\{\begin{array} { l } 
{ f _ { 1 } = \frac { ( r + 2 \delta ) k - \sqrt { ( r + 2 \delta ) ^ { 2 } k ^ { 2 } - \beta k \Delta ^ { 2 } \rho ^ { 2 } } } { 2 \rho ^ { 2 } } } \\
{ f _ { 2 } = \frac { k } { 4 } \frac { \Delta ( \phi - \beta c _ { m } ) + 2 \sigma ^ { 2 } f _ { 1 } } { ( r + \delta ) k - \rho ^ { 2 } f _ { 1 } } } \\
{ f _ { 3 } = \frac { k ( \phi - \beta c _ { m } ) ^ { 2 } + 4 \beta \rho ^ { 2 } f _ { 2 } ^ { 2 } } { 8 \beta k r } }
\end{array} \left\{\begin{array}{l}
g_{1}=\frac{1}{8} \frac{\beta k \Delta^{2}}{(r+2 \delta) k-2 \rho^{2} f_{1}} \\
g_{2}=\frac{1}{8} \frac{\Delta k\left(\phi-\beta c_{m}\right)+4 k \sigma^{2} g_{1}+8 \rho^{2} g_{1} f_{2}}{(r+\delta) k-\rho^{2} f_{1}} \\
g_{3}=\frac{k\left(\phi-\beta c_{m}\right)^{2}+16 \beta \rho^{2} g_{2} f_{2}}{16 \beta k r}
\end{array}\right.\right.
$$

The assumption $4 \delta(r+\delta) k-\beta \Delta^{2} \rho^{2}>0$ in Proposition 2, which is similar to the one in the integrated channel scenario, indicates that the return cost coefficient $k$ is sufficiently large, i.e., $k>\beta \Delta^{2} \rho^{2} / 4 \delta(r+\delta)$, so that the manufacturer has no motivation to return all used products. This result is quite similar to the results for the static model of a CLSC [11]. In practice, it is hardly possible to collect all units of the used products for the manufacturer. The reasons may include the wide and disordered distribution of used products, high transportation expenses, and the preference of some customers for keeping the old products.

Table 1 provides the comparative statics for the equilibrium strategies on system parameters. This table demonstrates that an increase of cost savings resulting from remanufacturing would result in a decrease of the retail price of the same product, along with an increase in the return effort of the manufacturer, all of which are beneficial to both the supply chain members and the customers. When the cost of production is on the rise, the manufacturer and the retailer would increase the wholesale price and retail price, and the manufacturer would lower the level of return effort. Moreover, when the intensity of stochastic disturbance is increasing, the manufacturer would improve the level of return effort. However, the prices of both the manufacturer and the retailer would not be affected by the stochastic disturbance.

Table 1. Comparative statics results for the equilibrium strategies.

\begin{tabular}{cccc}
\hline \multirow{2}{*}{ Equilibrium Strategies } & \multicolumn{3}{c}{ Parameters } \\
\cline { 2 - 4 } & $\Delta$ & $C_{m}$ & $\sigma$ \\
\hline$w^{D^{*}}$ & $\downarrow$ & $\uparrow$ & - \\
$P^{D^{*}}$ & $\downarrow$ & $\uparrow$ & - \\
$A^{D^{*}}$ & $\uparrow$ & $\downarrow$ & $\uparrow$ \\
\hline
\end{tabular}

Notes: increasing $(\uparrow)$, decreasing $(\downarrow)$, unchanged (-). 
4.2. The Evolutionary Path of the Stochastic Return Rate

Inserting the optimal control strategies in Proposition 2 into the system dynamics equation, Equation (2), we can show the following:

$$
d \tau=\left(-\left(\delta-\frac{\rho^{2} f_{1}}{k}\right) \tau+\frac{\rho^{2} f_{2}}{k}\right) d t+\sigma \sqrt{\tau} d z
$$

Since $(r+2 \delta)^{2} k^{2}-\beta k \Delta^{2} \rho^{2}>r^{2} k^{2}$, we have the equation below:

$$
\delta-\frac{\rho^{2} f_{1}}{k}=\frac{-r k+\sqrt{(r+2 \delta)^{2} k^{2}-\beta k \rho^{2} \Delta^{2}}}{2 k}>0 .
$$

Denoting $\xi=\left(\delta-\frac{\rho^{2} f_{1}}{k}\right)$ and $\psi=\frac{\rho^{2} f_{2}}{k}$, Equation (29) becomes the following:

$$
d \tau=(-\xi \tau+\psi) d t+\sigma \sqrt{\tau} d z
$$

Rewriting Equation (29) as the stochastic integral equation, we get:

$$
\tau(t)=\tau_{0}+\int_{0}^{t}(-\xi \tau(s)+\psi) d s+\int_{0}^{t} \sigma \sqrt{\tau(s)} d z(s) .
$$

Taking the expectation of the above equation, we get:

$$
E[\tau(t)]=\tau_{0}+\int_{0}^{t}(-\xi E[\tau(s)]+\psi) d s
$$

The above can be seen as an ordinary differential equation in $E(\tau(t))$ with $E(\tau(0))=\tau_{0}$. Solving the equation, we have the following result:

$$
E[\tau(t)]=\frac{\psi}{\xi}+\left(\tau_{0}-\frac{\psi}{\xi}\right) e^{-\tau t} .
$$

Taking the limit as $t \rightarrow \infty$, we can obtain the long-run stable return rate:

$$
\lim _{t \rightarrow \infty} E[\tau(t)]=\frac{\psi}{\xi}
$$

To guarantee that the long-run stable return rate is smaller than 1 , we assume that $\xi \geq \psi$, i.e., $k \geq \rho^{2}\left(f_{1}+f_{2}\right) / \delta$. To solve the variance of return rate, apply the Itô formula to Equation (29):

$$
d \tau^{2}=\left[2 \tau(-\xi \tau+\psi)+\sigma^{2} \tau\right] d \tau+2 \tau \sigma \sqrt{\tau} d z
$$

We rewrite the result as the stochastic integral equation:

$$
\tau^{2}(t)=\tau_{0}^{2}+\int_{0}^{t}\left[\left(2 \psi+\sigma^{2}\right) \tau(s)-2 \xi \tau^{2}(s)\right] d s+\int_{0}^{t} 2 \tau(s) \sigma \sqrt{\tau(s)} d z(s) .
$$

Then we take the expected value:

$$
E\left[\tau^{2}(t)\right]=\tau_{0}^{2}+\int_{0}^{t}\left[\left(2 \psi+\sigma^{2}\right) E[\tau(s)]-2 \xi E\left[\tau^{2}(s)\right]\right] d s .
$$


Inserting the expression of $E[\tau(t)]$ in Equation (32), and solving the linear differential equation, we get the following:

$$
E\left[\tau^{2}(t)\right]=\frac{\psi\left(2 \psi+\sigma^{2}\right)}{2 \xi^{2}}\left(1-e^{-2 \xi t}\right)+\frac{\left(\tau_{0} \xi-\psi\right)\left(2 \psi+\sigma^{2}\right)}{\xi^{2}}\left(e^{-\xi^{2} t}-e^{-2 \xi t}\right)+\tau_{0}^{2} e^{-2 \xi t} .
$$

Therefore,

$$
D[\tau(t)]=E\left[\tau^{2}(t)\right]-[E[\tau(t)]]^{2}=\frac{\psi \sigma^{2}}{2 \xi^{2}}+\frac{\left(\tau_{0} \xi-\psi\right) \sigma^{2}}{\xi^{2}} e^{-\xi t}-\frac{\left(2 \tau_{0} \xi-\psi\right) \sigma^{2}}{2 \xi^{2}} e^{-2 \xi t} .
$$

Using a similar approach, we can find the stable value of the variance of the return rate.

Proposition 3. The expected value and variance of return rate are calculated by the following:

$$
\begin{gathered}
E[\tau(t)]=\frac{\psi}{\xi}+\left(\tau_{0}-\frac{\psi}{\xi}\right) e^{-\xi t}, \\
D[\tau(t)]=\frac{\psi \sigma^{2}}{2 \xi^{2}}+\frac{\left(\tau_{0} \xi-\psi\right) \sigma^{2}}{\xi^{2}} e^{-\xi t}-\frac{\left(2 \tau_{0} \xi-\psi\right) \sigma^{2}}{2 \xi^{2}} e^{-2 \xi t} .
\end{gathered}
$$

The long-run stable expected and variance values of the return rate are calculated:

$$
\lim _{t \rightarrow \infty} E[\tau(t)]=\frac{\psi}{\xi}, \lim _{t \rightarrow \infty} D[\tau(t)]=\frac{\psi \sigma^{2}}{2 \xi^{2}} .
$$

Proposition 3 indicates that the expectation and variance of the return rate are convergent. From the expected value of the return rate, we have:

$$
d \frac{E[\tau(t)]}{d t}=-\xi\left(\tau_{0}-\frac{\psi}{\xi}\right) e^{-\xi t}
$$

Hence, when $\xi \tau_{0}-\psi>0$, i.e., $\tau_{0}>\psi / \xi$, then $d E[\tau(t)] / d t<0$; otherwise, when $\xi \tau_{0}-\psi<0$, i.e., $\tau_{0}<\psi / \xi$, then $d E[\tau(t)] / d t>0$. Similarly,

$$
d \frac{D[\tau(t)]}{d t}=\frac{\sigma^{2}}{\xi^{2}}\left(\left(2 \tau_{0} \xi-\psi\right)-\left(\tau_{0} \xi-\psi\right) e^{-\xi t}\right)
$$

when $\xi \tau_{0}-\psi<0$, it is obvious that $d D[\tau(t)] / d t>0$. On the contrary, when $\xi \tau_{0}-\psi>0$, on condition that $\left(2 \tau_{0} \xi-\psi\right)-\left(\tau_{0} \xi-\psi\right) e^{-\xi t}>0$, i.e., $0<t<\ln \frac{2 \tau_{0} \xi-\psi}{\xi\left(\tau_{0} \xi-\psi\right)}$, then $d D[\tau(t)] / d t>0$, otherwise on condition that $\left(2 \tau_{0} \xi-\psi\right)-\left(\tau_{0} \xi-\psi\right) e^{-\xi t}<0$, i.e., $\ln \frac{2 \tau_{0} \xi-\psi}{\xi\left(\tau_{0} \xi-\psi\right)}<t$, then $d D[\tau(t)] / d t<0$.

\section{Proposition 4.}

(1) When $\sigma=0, \lim _{t \rightarrow \infty} E\left[(\tau(t))^{2}\right]=\left[\lim _{t \rightarrow \infty} E[\tau(t)]\right]^{2}$, and $\lim _{t \rightarrow \infty} D[\tau(t)]=0$.

(2) When $\tau_{0}>\psi / \xi$, the expected value of the return rate decreases with time; otherwise, when $\tau_{0}<\psi / \xi$, the expected value of the return rate increases with time.

(3) When $\tau_{0}<\psi / \xi$, the variance of the return rate increases with time; otherwise, when $\tau_{0}>\psi / \xi$, the variance of the return rate increases first at $0<t<\ln \frac{2 \tau_{0} \xi-\psi}{\xi\left(\tau_{0} \xi-\psi\right)}$, and then decreases with time when $t>\ln \frac{2 \tau_{0} \xi-\psi}{\xi\left(\tau_{0} \xi-\psi\right)}$.

Proposition 4 investigates the evolution of the expectation and variance of the return rate, which indicates that the expectation and variance is stable from a long-run perspective. However, as a result of the stochastic disturbance, the real return rate would deviate from the expected value. 
In the following, we utilize the methods used by Prasad and Sethi [41] to investigate the disturbance of stochastic factors on the return rate.

We use the following method to approximate the differential equation in Equation (2):

$$
\tau(t+\Delta t)=\tau(t)+(-\xi \tau(t)+\psi) \Delta t+\sigma \sqrt{\tau(t)} \sqrt{\Delta t} \zeta(t),
$$

where $\{\zeta(t)\}$ are independent and identically distributed (i.i.d.) standard normal random variables. We set the time step $\Delta t$ to be 0.01 . The others parameters are chosen by: $\phi=50, \beta=0.8, c_{m}=6, \Delta=2$, $\rho=2, \delta=1, r=0.15, k=230, \sigma=0.5$, and $\tau_{0}=0$. Figure 1 gives the simulation result.

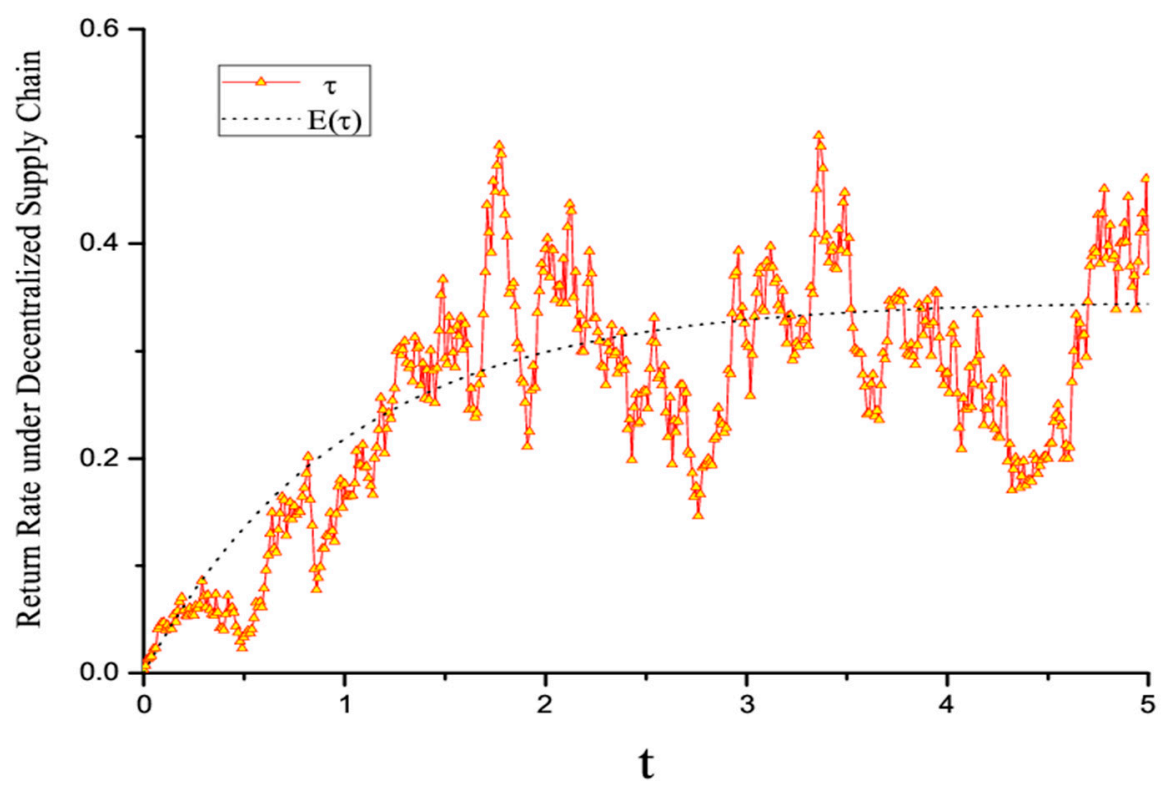

Figure 1. The simulation of the stochastic return rate of decentralized closed-loop supply chain (CLSC).

As can be seen from Figure 1, a result of the stochastic disturbance is that the return rate is always deviating from the expected value. However, it should be noted that the return rate always hovers around its expectation. The expected return rate would be stable when the time is sufficient long, however, even if the expected return rate is stable the real return rate would not be stable as the disturbance of the stochastic factors in the collection process. Assuming that the return rate is normally distributed around the expected value, the confidence interval at the $95 \%$ level can be calculated as shown:

$$
(E[\tau(t)]-1.96 \sqrt{D[\tau(t)]}, E[\tau(t)]+1.96 \sqrt{D[\tau(t)]}) .
$$

\subsection{The Probability Distribution of the Stochastic Return Rate}

In Section 4.2, we assumed the normal distribution to calculate the confidence interval for the return rate. However, this approach is just a better approximation in many cases. In this subsection, we attempt to deduce the probability distribution of the return rate.

The solution of Equation (29) is a Markov process. We denote the transition probability of the return rate from time $s$ return rate $\omega$, to time, $t>s$ return rate $\tau$, as $f(t, \tau ; s, \omega)$, which should satisfy the Fokker-Planck equation,

$$
\frac{\partial f}{\partial t}+\frac{\partial((-\xi \tau+\psi) f)}{\partial \tau}-\frac{1}{2} \frac{\partial^{2}\left(\sigma^{2} \tau f\right)}{\partial \tau^{2}}=0
$$


The solution of the above partial differential equation is the probability density function. However, the partial differential equation is hard to solve explicitly. Instead, we could solve the stable transition probability density $\lim _{t \rightarrow \infty} f(t, \tau ; s, \omega)$. It is obvious that $f(t, \tau ; s, \omega)$ now is independent of $t, s, \omega$, and only dependent on $\tau$. Denoting it as $f(\tau)$, since $\partial f / \partial t=0$, we have the following equation:

$$
\tau f^{\prime \prime}(\tau)+\left(\frac{2 \xi}{\sigma^{2}} \tau+\frac{2\left(\sigma^{2}-\psi\right)}{\sigma^{2}}\right) f^{\prime}(\tau)+\frac{2 \xi}{\sigma^{2}} f(\tau)=0 .
$$

The solution of the above second order non-autonomous differential equation is the probability density of the return rate.

Proposition 5. On condition that the system is stable, the probability density of the return rate can be calculated by the following equation:

$$
f(\tau)=\tau^{1-v} e^{-u t}\left[C_{1}+C_{2}\left((-u)^{-(v+1)}(\Gamma(v+1)-v \Gamma(v,-u \tau))+\frac{\tau^{v}}{u} e^{u \tau}\right)\right],
$$

where $u=\frac{2 \xi}{\sigma^{2}}, v=\frac{2\left(\sigma^{2}-\psi\right)}{\sigma^{2}}, \Gamma(s)=\int_{0}^{\infty} x^{s-1} e^{-x} d x$, and $\Gamma(s, a)=\int_{a}^{\infty} x^{s-1} e^{-x} d x$. The undetermined coefficients $C_{1}$ and $C_{2}$ can be solved by the conditions $\int_{0}^{1} f(\tau)=1$ and $\int_{0}^{1} \tau f(\tau)=\frac{\psi}{\xi}$.

\section{Channel Coordination}

In this section, we study channel coordination in the decentralized CLSC, and show that the stochastic supply chain can be coordinated by our proposed contract. Assume the contract provided by the manufacturer to the retailer is $\left(F, w^{S C}\right)$, where $F$ represents the franchise fee paid by the retailer to the manufacturer for the sale of the product, and $w^{S C}$ is the wholesale price in the coordination model. The retailer decides whether to take the contract or not, and the manufacturer's problem can be formulated by the principal-agent theory as follows:

$$
\begin{aligned}
& \max _{w^{S C}>0, A^{S C}>0}\left\{J_{m}^{S C}=E \int_{0}^{\infty} e^{-r t}\left[\left(w^{S C}-c_{m}+\Delta \tau\right)\left(\phi-\beta p^{S C}\right)-\frac{k}{2}\left(A^{S C}\right)^{2}+F\right] d t\right\} \\
& \text { s.t. }\left\{\begin{array}{l}
p^{S C}=\underset{\operatorname{argmax}}{S C *}\left\{J_{r}^{S C}=E \int_{0}^{\infty} e^{-r t}\left[\left(p^{S C}-w^{S C}\right)\left(\phi-\beta p^{S C}\right)-F\right] d t\right\} \\
J_{r}^{S C *}>r V_{r}^{D *}
\end{array},\right.
\end{aligned}
$$

where the first constraint is an incentive compatible constraint, and the second constraint is an individual rationality constraint. The profit under the coordinated scenario should not be less than the profit under the decentralized scenario, or else the retailer would never choose the contract. Proposition 6 gives the expression of the contract that can coordinate the CLSC.

Proposition 6. Under the coordination model, the wholesale price and level of collection effort of the manufacturer is calculated as follows:

$$
w^{S C *}=c_{m}-\Delta \tau, A^{S C *}=\frac{\rho}{k}\left(e_{1} \tau+e_{2}\right) .
$$

The retail price of the retailer is calculated as shown:

$$
p^{S C *}=\frac{\phi+\beta c_{m}-\beta \Delta \tau}{2 \beta} .
$$


The value function of the manufacturer and retailer is calculated by the following:

$$
\left\{\begin{array}{l}
V_{r}^{S C *}=\frac{1}{2} g_{1} \tau^{2}+g_{2} \tau+g_{3} \\
V_{m}^{S C *}=\frac{1}{2}\left(e_{1}-g_{1}\right) \tau^{2}+\left(e_{2}-g_{2}\right) \tau+\left(e_{3}-g_{3}\right)
\end{array} .\right.
$$

The franchise fee is calculated by this next equation:

$$
F=\left\{\begin{array}{l}
\left(\frac{1}{4} \beta \Delta^{2}-\delta g_{1}-\frac{1}{2} r g_{1}+\frac{\rho^{2} g_{1} e_{1}}{k}\right) \tau^{2} \\
\left(+\frac{1}{2} \Delta\left(\phi-\beta c_{m}\right)-(r+\delta) g_{2}+\frac{1}{2} g_{1} \sigma^{2}+\frac{\rho^{2}\left(g_{2} e_{1}+g_{1} e_{2}\right)}{k}\right) \tau \\
\left(+\frac{\left(\phi-\beta c_{m}\right)^{2}}{4 \beta}-r g_{3}+\frac{\rho^{2} g_{2} e_{2}}{k}\right)
\end{array}\right\},
$$

where the expressions of $e_{i}$ and $g_{i}(i=1,2,3)$ are given in Proposition 1 and Proposition 2.

Proof. The HJB equation of the retailer is

$$
r V_{r}=\max _{p^{S C}}\left\{\left(p^{S C}-w^{S C}\right)\left(\phi-\beta p^{S C}\right)+V^{\prime}{ }_{r}(\tau)\left(\rho A^{S C}-\delta \tau\right)+\frac{\sigma^{2}}{2} \tau V^{\prime \prime}{ }_{r}(\tau)-F\right\} .
$$

According to the first order condition, we get $p^{S C *}=\left(\phi+\beta w^{S C}\right) / 2 \beta$, and from

$$
r V_{r}=\left(p^{S C *}-w^{S C}\right)\left(\phi-\beta p^{S C *}\right)+V^{\prime}{ }_{r}(\tau)\left(\rho A^{S C}-\delta \tau\right)+\frac{\sigma^{2}}{2} \tau V^{\prime \prime}{ }_{r}(\tau)-F \geq r V_{r}^{D *}
$$

we derive $F \leq\left(p^{S C *}-w^{S C}\right)\left(\phi-\beta p^{S C *}\right)+V^{\prime}{ }_{r}(\tau)\left(\rho A^{S C}-\delta \tau\right)+\frac{\sigma^{2}}{2} \tau V^{\prime \prime}{ }_{r}(\tau)-r V_{r}^{D *}$. Thus, the HJB equation of the manufacturer is

$$
r V_{m}=\max _{w^{S C}}\left\{\begin{array}{l}
\left(w^{S C}-c_{m}+\Delta \tau\right)\left(\phi-\beta p^{S C *}\right)-\frac{k}{2}\left(A^{S C}\right)^{2}+V^{\prime}{ }_{m}(\tau)\left(\rho A^{S C}-\delta \tau\right)+\frac{\sigma^{2}}{2} \tau V^{\prime \prime}{ }_{m}(\tau) \\
+\left(p^{S C *}-w^{S C}\right)\left(\phi-\beta p^{S C *}\right)+V^{\prime}{ }_{r}(\tau)\left(\rho A^{S C}-\delta \tau\right)+\frac{\sigma^{2}}{2} \tau V^{\prime \prime}{ }_{r}(\tau)-r V_{r}^{D *}
\end{array}\right\} .
$$

According to the first order condition, we resolve the optimal collection and wholesale price control strategy:

$$
A^{s c *}=\frac{\rho}{k}\left(V^{\prime}{ }_{m}+V_{r}^{\prime}\right), w^{S C *}=c_{m}-\Delta \tau .
$$

Taking back the above equation into the retailer's reaction function, we resolve the optimal retail price control strategy:

$$
p^{S C *}=\frac{\phi+\beta c_{m}-\beta \Delta \tau}{2 \beta} .
$$

Taking the optimal control strategies of the manufacturer and the retailer into their value functions, we get the following result:

$$
\begin{gathered}
r V_{r}=r V_{r}^{D *} \\
r V_{m}=\left\{\begin{array}{l}
\frac{1}{4} \beta \Delta^{2} \tau^{2}+\left(\frac{1}{2} \Delta\left(\phi-\beta c_{m}\right)-\delta\left(V^{\prime}{ }_{m}+V_{r}^{\prime}\right)+\frac{1}{2} \sigma^{2}\left(V^{\prime \prime}{ }_{m}+V^{\prime \prime}{ }_{r}\right)\right) \tau \\
+\frac{\left(\phi-\beta c_{m}\right)^{2}}{4 \beta}+\frac{1}{2} \frac{\rho^{2}}{k}\left(V_{m}^{\prime}{ }_{m}+V_{r}^{\prime}\right)^{2}-r V_{r}^{D *}
\end{array}\right\} .
\end{gathered}
$$

It is clear that

$$
r\left(V_{m}+V_{r}\right)=\left\{\begin{array}{l}
\frac{1}{4} \beta \Delta^{2} \tau^{2}+\left(\frac{1}{2} \Delta\left(\phi-\beta c_{m}\right)-\delta\left(V^{\prime}{ }_{m}+V^{\prime}{ }_{r}\right)+\frac{1}{2} \sigma^{2}\left(V^{\prime \prime}{ }_{m}+V^{\prime \prime}{ }_{r}\right)\right) \tau \\
+\frac{\left(\phi-\beta c_{m}\right)^{2}}{4 \beta}+\frac{1}{2} \frac{\rho^{2}}{k}\left(V_{m}^{\prime}{ }_{m}+V^{\prime}{ }_{r}\right)^{2}
\end{array}\right\} .
$$




$$
\begin{gathered}
\text { Assuming }\left\{\begin{array} { l } 
{ V _ { r } = \frac { 1 } { 2 } l _ { 1 } \tau ^ { 2 } + l _ { 2 } \tau + l _ { 3 } } \\
{ V _ { m } = \frac { 1 } { 2 } m _ { 1 } \tau ^ { 2 } + m _ { 2 } \tau + m _ { 3 } }
\end{array} \text { , then } \left\{\begin{array}{l}
V_{r}^{\prime}+V_{m}^{\prime}=\left(l_{1}+m_{1}\right) \tau+l_{2}+m_{2}, \text { calculate as } \\
V_{r}^{\prime \prime}+V^{\prime \prime}{ }_{m}=\left(l_{1}+m_{1}\right)
\end{array}\right.\right. \\
\left\{\begin{array}{l}
l_{1}+m_{1}=\frac{(r+2 \delta) k-\sqrt{(r+2 \delta)^{2} k^{2}-2 \beta k \rho^{2} \Delta^{2}}}{2 \rho^{2}}=e_{1} \\
l_{2}+m_{2}=\frac{1}{2} \frac{\left[\left(\phi-\beta c_{m}\right) \Delta+\sigma^{2}\left(l_{1}+m_{1}\right)\right] k}{(r+\delta) k-\rho^{2}\left(l_{1}+m_{1}\right)}=e_{2} \\
l_{3}+m_{3}=\frac{k\left(\phi-\beta c_{m}\right)^{2}+2 \rho^{2} \beta\left(l_{2}+m_{2}\right)^{2}}{4 \beta k r}=e_{3}
\end{array}\right.
\end{gathered}
$$

where $l_{1}=g_{1}, l_{2}=g_{2}, l_{3}=g_{3}$. Then $m_{1}=e_{1}-l_{1}, m_{2}=e_{2}-l_{2}, m_{3}=e_{3}-l_{3}$.

Taking back the derivatives of the calculated value functions into the optimal strategies, we can obtain the expressions in Proposition 6. The proof is complete.

Proposition 6 indicates that the manufacturer applies the marginal cost pricing under the coordination model. This action is quite likely in the two-part tariffs scenario [42-45]. It is important that the retailer applied the marginal pricing under the coordination model, which avoided the double marginalization problem in the decentralized CLSC. The retailer transfers the profit to the manufacturer by the franchise fee. As a result of remanufacturing, the actual production cost turned to the unit cost minus the cost savings brought by the used-product collecting. The manufacturer also applied the marginal pricing, taking the actual production cost. The retail price and the collection effort level are the same as under the centralized model. The reason for the low level of recycling investment in the decentralized supply chain is that the level of the collection effort does not reflect the marginal value of product recovery to the other member of the supply chain. When the manufacturer considers only the marginal value of product recycling, obviously her enthusiasm for the investment will be reduced. However, in the coordination model, this situation has been avoided, both manufacturer and retailer had the motivation to lower the cost by raising the return and the remanufacturing CLSC can reach the level of the centralized supply chain.

\section{Conclusions}

For a remanufacturing CLSC, the basic procedure is to collect used products to be recycled into the production of new products. In this paper, we considered the impact of stochastic disturbance on the dynamic collection process in a CLSC consisting of a manufacturer and a retailer, where the manufacturer is responsible for collecting the used products. We also investigated the coordination problem faced by a decentralized CLSC.

First, we proposed a dynamic collection equation that considered the stochastic disturbance. We used a stochastic collection model based on the Itô equation, and then we derived the optimal feedback control strategies for both the manufacturer and retailer in centralized and decentralized system settings, respectively. The evolutionary path and probability distribution of the stochastic return rate were analyzed. Finally, we proposed a contract to coordinate the decentralized supply chain.

The optimal control strategy was derived using the HJB equation method. The results of comparative statistics for the equilibrium strategies showed that increasing cost savings from remanufacturing would result in increasing the return effort by the manufacturer. Furthermore, increasing cost savings from remanufacturing would result in decreasing retailer pricing of the product. In contrast, when the cost of production is increasing, the manufacturer and the retailer would increase the wholesale price and retail price, and the manufacturer would lower the level of return effort. We demonstrated that in either case, when the stochastic disturbance intensity is increasing, the manufacturer would improve the level of return effort. However, neither manufacturer nor retailer pricing would be affected by the stochastic disturbance. All of the above changes are beneficial to both the supply chain members and the customers.

As a result of stochastic disturbance, the return rate always hovered around the expected return rate. However, the expectation and variance of the return rate proved to be stable from a long-term 
perspective. The expected value of the return rate and the variance might decrease or increase with time, depending on the value of the initial return rate of the system.

The proposed contract could coordinate the stochastic supply chain as the manufacturer utilizes the marginal cost pricing under the coordination model. The actual production cost equals the unit cost minus the cost savings brought by the collection of used products.

It is interesting from the comparative statics results in Table 1 that the stochastic disturbance would not affect the strategies of forward channel. However, the table demonstrates that the cost savings as well as the production cost would affect both the forward decision and the reverse decision. This means even if the stochastic disturbance is intensive, it would not transmit to the forward channel.

It should be noticed that the stable return rate is solved by taking the time limit as $t \rightarrow \infty$; however, for a real CLSC system, it is unrealistic that the time would go for infinity. As a result, we should keep in mind that the real return rate would not be stable as the disturbance of the stochastic factors in the collection process, which is shown in Figure 1.

The equilibrium of both integrated channel as well as the decentralized channel exist only when the collection cost coefficient could not be too small. It is interesting that there is similar assumption in the static model [11]. It can be interpreted as the when the collection cost coefficient is too small, the manufacturer would have motivation to collect all the product while this cannot be true in practice. As a result of the wide and disordered distribution of used products as well as the special preference of some customers, the manufacturer cannot collect all used products. Consequently, the assumption is reliable from the perspective of reality.

To develop a more general understanding of the impact of stochastic disturbance on CLSCs, future efforts could extend our model to consider cases in which either the retailer or a third party collects the used products. The reverse channel design problem could be researched in the stochastic model as well. Furthermore, the competitive environment is also worth investigating, which would increase the mathematical difficulties.

Acknowledgments: This paper was supported in part by the National Natural Science Foundation of China with Grants Nos. 71602116, 71672153 and 71571117; the Innovation Ability Construction Projects for Shanghai University through Grant Nos. 15590501800; the Academic Scientific Research Foundation for High-Level Researchers, University of Electronic Science Technology of China, Zhongshan Institute (No. 415YKQ08); the Tianjin Philosophy and Social Science Planning Project (No. TJGL13-028); and the Fundamental Research Funds for the Central Universities (No. ZXH2012N002).

Author Contributions: Writing: Zongsheng Huang, Jiajia Nie; Providing case and idea: Zongsheng Huang, Jiajia Nie; Providing revised advice: Zongsheng Huang, Sang-Bing Tsai.

Conflicts of Interest: The authors declare no conflict of interest.

\section{References}

1. Qiang, Q. The closed-loop supply chain network with competition and design for remanufactureability. J. Clean. Prod. 2015, 105, 348-356. [CrossRef]

2. Toffel, M.W. Strategic management of product recovery. Calif. Manag. Rev. 2004, 46, 120-141. [CrossRef]

3. Savaskan, R.C.; Wassenhove, L.N.V. Reverse channel design: The case of competing retailers. Manag. Sci. 2006, 52, 1-14. [CrossRef]

4. Qiang, Q.; Ke, K.; Anderson, T.; Dong, J. The closed-loop supply chain network with competition, distribution channel investment and uncertainties. Omega 2013, 41, 186-194. [CrossRef]

5. Shaharudin, M.R.; Govindan, K.; Zailani, S.; Tan, K.C.; Iranmanesh, M. Product return management: Linking product returns, closed-loop supply chain activities and the effectiveness of the reverse supply chains. J. Clean. Prod. 2017, 149, 1144-1156. [CrossRef]

6. Özkır, V.; Başlıgil, H. Multi-objective optimization of closed-loop supply chains in uncertain environment. J. Clean. Prod. 2013, 41, 114-125. [CrossRef]

7. Shi, J.; Zhang, G.; Sha, J. Optimal production planning for a multi-product closed loop system with uncertain demand and return. Comput. Oper. Res. 2011, 38, 641-650. [CrossRef] 
8. Giri, B.C.; Sharma, S. Optimal production policy for a closed-loop hybrid system with uncertain demand and return under supply disruption. J. Clean. Prod. 2015, 112, 2015-2028. [CrossRef]

9. Shaharudin, M.R.; Govindan, K.; Zailani, S.; Tan, K.C. Managing product returns to achieve supply chain sustainability: An exploratory study and research propositions. J. Clean. Prod. 2015, 101, 1-15. [CrossRef]

10. Heydari, J.; Govindan, K.; Jafari, A. Reverse and closed loop supply chain coordination by considering government role. Transp. Res. 2017, 52, 379-398. [CrossRef]

11. Savaskan, R.C.; Bhattacharya, S.; van Wassenhove, L.N. Closed-loop supply chain models with product remanufacturing. Manag. Sci. 2004, 50, 239-252. [CrossRef]

12. de Giovanni, P.; Zaccour, G. Cost-Revenue Sharing in a Closed-Loop Supply Chain. In Advances in Dynamic Games: Theory, Applications, and Numerical Methods for Differential and Stochastic Games; Cardaliaguet, P., Cressman, R., Eds.; Birkhäuser Boston: Boston, MA, USA, 2013; pp. 395-421.

13. Nerlove, M.; Arrow, K. Optimal advertising policy under dynamic conditions. Economica 1962, $29,129-142$. [CrossRef]

14. Vidale, M.L.; Wolfe, H.B. An operations-research study of sales response to advertising. Oper. Res. 1957, 5, 370-381. [CrossRef]

15. Bass, F.M. A new product growth model for consumer durables. Manag. Sci. 1969, 15, 215-227. [CrossRef]

16. Jørgensen, S.; Zaccour, G. A survey of game-theoretic models of cooperative advertising. Eur. J. Oper. Res. 2014, 237, 1-14. [CrossRef]

17. Aust, G.; Buscher, U. Cooperative advertising models in supply chain management: A review. Eur. J. Oper. Res. 2014, 234, 1-14. [CrossRef]

18. Qu, Q.; Tsai, S.-B.; Tang, M.; Xu, C.; Dong, W. Marine Ecological Environment Management Based on Ecological Compensation Mechanisms. Sustainability 2016, 8, 1267. [CrossRef]

19. Huang, Z.; Nie, J. Dynamic closed-loop supply chain model with product remanufacturing. In Proceedings of the 2012 International Conference of Logistics Engineering and Management, Chengdu, China, 8-10 October 2012; pp. 1039-1045.

20. Atasu, A.; Sarvary, M.; Wassenhove, L.N.V. Remanufacturing as a marketing strategy. Manag. Sci. 2008, 54, 1731-1746. [CrossRef]

21. Govindan, K.; Soleimani, H.; Kannan, D. Reverse logistics and closed-loop supply chain: A comprehensive review to explore the future. Eur. J. Oper. Res. 2015, 240, 603-626. [CrossRef]

22. Govindan, K.; Soleimani, H. A review of reverse logistics and closed-loop supply chains: A journal of cleaner production focus. J. Clean Prod. 2017, 142, 371-384. [CrossRef]

23. Huang, M.; Song, M.; Lee, L.H.; Ching, W.K. Analysis for strategy of closed-loop supply chain with dual recycling channel. Int. J. Prod. Econ. 2013, 144, 510-520. [CrossRef]

24. Zhang, Z.Z.; Wang, Z.J.; Liu, L.W. Retail services and pricing decisions in a closed-loop supply chain with remanufacturing. Sustainability 2015, 7, 2373-2396. [CrossRef]

25. De Giovanni, P.; Zaccour, G. A two-period game of a closed-loop supply chain. Eur. J. Oper. Res. 2014, 232, 22-40. [CrossRef]

26. Tsai, S.B.; Lee, Y.C.; Guo, J.J. Using modified grey forecasting models to forecast the growth trends of green materials. Proc. Inst. Mech. Eng. J. Eng. Manuf. 2014, 228, 931-940. [CrossRef]

27. Lee, Y.C.; Chu, W.H.; Chen, Q.; Tsai, S.B.; Wang, J.; Dong, W. Integrating DEMATEL Model and Failure Mode and Effects Analysis to Determine the Priority in Solving Production Problems. Adv. Mech. Eng. 2016, 8, 1-12. [CrossRef]

28. Su, J.M.; Lee, S.C.; Tsai, S.B.; Lu, T.L. A comprehensive survey of the relationship between self-efficacy and performance for the governmental auditors. SpringerPlus 2016, 5, 508. [CrossRef] [PubMed]

29. Tsai, S.B.; Chen, K.Y.; Zhao, H.; Wei, Y.M.; Wang, C.K.; Zheng, Y.; Chang, L.C.; Wang, J. Using a Mixed Model to Explore Evaluation Criteria for Bank Supervision: A Banking Supervision Law Perspective. PLoS ONE 2016, 11, e0167710. [CrossRef] [PubMed]

30. Zhou, J.; Wang, Q.; Tsai, S.B.; Xue, Y.; Dong, W. How to Evaluate the Job Satisfaction of Development Personnel. IEEE Trans. Syst. Man Cybern. Syst. 2016. [CrossRef]

31. Subramanian, R.; Gupta, S.; Talbot, B. Product design and supply chain coordination under extended producer responsibility. Prod. Oper. Manag. 2009, 18, 259-277. [CrossRef]

32. Jacobs, B.W.; Subramanian, R. Sharing responsibility for product recovery across the supply chain. Prod. Oper. Manag. 2011, 21, 85-100. [CrossRef] 
33. Jena, S.K.; Sarmah, S.P. Price competition and co-operation in a duopoly closed-loop supply chain. Int. J. Prod. Econ. 2014, 156, 346-360. [CrossRef]

34. Ma, Z.J.; Zhang, N.; Dai, Y.; Hu, S. Managing channel profits of different cooperative models in closed-loop supply chains. Omega 2015, 59, 251-262.

35. Guide, V.D.R.; Wassenhove, L.N. Managing product returns for remanufacturing. Prod. Oper. Manag. 2001, 10, 142-155. [CrossRef]

36. Nakashima, K.; Arimitsu, H.; Nose, T.; Kuriyama, S. Optimal control of a remanufacturing system. Int. J. Prod. Res. 2004, 42, 3619-3625. [CrossRef]

37. Fallah, H.; Eskandari, H.; Pishvaee, M.S. Competitive closed-loop supply chain network design under uncertainty. J. Manuf. Sys. 2015, 37, 649-661. [CrossRef]

38. Krikke, H.; Le Blanc, I.; van de Velde, S. Product modularity and the design of closed-loop supply chains. Calif. Manag. Rev. 2004, 46, 23-39. [CrossRef]

39. Ge, B.; Jiang, D.; Gao, Y.; Tsai, S.B. The Influence of Legitimacy on a Proactive Green Orientation and Green Performance: A Study Basedon Transitional Economy Scenarios in China. Sustainability 2016, 8, 1344. [CrossRef]

40. Xiong, Y.; Li, G.; Zhou, Y.; Fernandes, K.; Harrison, R.; Xiong, Z. Dynamic pricing models for used products in remanufacturing with lost-sales and uncertain quality. Int. J. Prod. Econ. 2014, 147, 678-688. [CrossRef]

41. Prasad, A.; Sethi, S.P. Competitive advertising under uncertainty: A stochastic differential game approach. J. Optim. Theory App. 2004, 123, 163-185. [CrossRef]

42. Erickson, G.M. A differential game model of the marketing-operations interface. Eur. J. Oper. Res. 2011, 211, 394-402. [CrossRef]

43. Moorthy, K.S. Managing channel profits: Comment. Mark. Sci. 1987, 6, 375-379. [CrossRef]

44. Borger, B.D. Optimal two-part tariffs in a model of discrete choice. J. Public Econ. 2000, 76, 127-150. [CrossRef]

45. Lee, Y.C.; Wu, C.H.; Tsai, S.B. Grey System Theory and Fuzzy Time Series Forecasting for the Growth of Green Electronic Materials. Int. J. Prod. Res. 2014, 299, 1395-1406. [CrossRef]

(C) 2017 by the authors. Licensee MDPI, Basel, Switzerland. This article is an open access article distributed under the terms and conditions of the Creative Commons Attribution (CC BY) license (http:/ / creativecommons.org/licenses/by/4.0/). 